\title{
Combining occurrence and abundance distribution models for the conservation of the Great Bustard
}

\author{
Chunrong Mi ${ }^{1}{ }$, Falk Huettmann ${ }^{2}{ }^{,}$Rui Sun ${ }^{3}$, Yumin Guo ${ }^{\text {Corresp. } 1}$ \\ ${ }^{1}$ College of Nature Conservation, Beijing Forestry University, Beijing, China \\ 2 EWHALE Lab, Department of Biology and Wildlife, Institute of Arctic Biology, University of Alaska - Fairbanks, Fairbanks, Alaska, United States \\ 3 Key Laboratory of Water Cycle and Related Land Surface Processes, Institute of Geographic Sciences and Natural Resources Research, University of \\ Chinese Academy of Sciences, Beijing, China \\ Corresponding Author: Yumin Guo \\ Email address: guoyumin@bjfu.edu.cn
}

Species distribution models (SDMs) have become important and essential tools in conservation and management. However, SDMs built with count data, commonly referred to as species abundance models (SAMs), are still less used to date. SDMs are increasingly being used now in conservation decisions, whereas SAMs are still not widely employed. Species occurrence and abundance do not frequently display similar patterns, and often they are not even well correlated. This leads to an insufficient or misleading conservation efforts. How to combine information from SDMs and SAMs and how to apply the combined information to achieve unified conservation remains a challenge. In this study, we introduce and propose a priority protection index (PI). The PI combines the prediction results of the occurrence and abundance models. As a case study, we used the bestavailable presence and count records for an endangered farmland species, the Great Bustard (Otis tarda dybowskii), in Bohai Bay, China, as a case study. We then applied the Random Forest algorithm (Salford Systems Ltd. Implementation), a powerful machine learning method, with eleven predictor variables to forecast the spatial occurrence as well as the abundance distribution. The results show that the occurrence model had a decent performance (ROC: 0.77 ) and the abundance model had a RMSE of 26.54. It is noteworthy that environmental variables influenced bustard occurrence and abundance differently. The area of farmland, and the distance to residential areas were the top important variables influencing bustard occurrence. In contrast, the distance to national roads and to expressways were the most important to variables influencing abundance. In addition, the occurrence and abundance models displayed different spatial distribution patterns. The regions with a high probability of occurrence were concentrated in the south-central part of the study area; and the abundance distribution showed high populations occurrence in the central and northwestern parts of the study area. Still, combining occurrence and abundance indices to produce a priority protection index $(\mathrm{PI})$ to be used for conservation 
could guide the protection of the areas with high occurrence and high abundance (e.g., in Strategic Conservation Planning). Due to the widespread use of SDMs and the easy subsequent employment of SAMs, these findings have a wide relevance and applicability. We promote and strongly encourage to further testing, application and updates to the priority protection index (PI) elsewhere to explore the generality of these findings and methods that are readily available now to researchers. 
1 Combining occurrence and abundance distribution models for the

3 Chunrong $\mathrm{Mi}^{1}$, Falk Huettmann ${ }^{2}$, Rui Sun ${ }^{3}$, Yumin Guo ${ }^{1, *}$

$4{ }^{1}$ College of Nature Conservation, Beijing Forestry University, Beijing, China;

$5 \quad{ }^{2}$ EWHALE Lab, Department of Biology and Wildlife, Institute of Arctic Biology, University of Alaska

6 Fairbanks (UAF), Alaska, USA

$7 \quad{ }^{3}$ Key Laboratory of Water Cycle and Related Land Surface Processes, Institute of Geographic Sciences

8 and Natural Resources Research, University of Chinese Academy of Sciences, Beijing, China

9 Corresponding author: Yumin Guo guoyumin@bjfu.edu.cn

10 College of Nature Conservation, Beijing Forestry University, P.O. Box 159, Beijing 100083, China 


\section{ABSTRACT}

12 Species distribution models (SDMs) have become important and essential tools in conservation

13 and management. However, SDMs built with count data, commonly referred to as species

14 abundance models (SAMs), are still less used to date. SDMs are increasingly being used now in

15 conservation decisions, whereas SAMs are still not widely employed. Species occurrence and

16 abundance do not frequently display similar patterns, and often they are not even well correlated.

17 This leads to an insufficient or misleading conservation efforts. How to combine information

18 from SDMs and SAMs and how to apply the combined information to achieve unified

19 conservation remains a challenge. In this study, we introduce and propose a priority protection

20 index (PI). The PI combines the prediction results of the occurrence and abundance models. As a

21 case study, we used the best-available presence and count records for an endangered farmland

22 species, the Great Bustard (Otis tarda dybowskii), in Bohai Bay, China, as a case study. We then

23 applied the Random Forest algorithm (Salford Systems Ltd. Implementation), a powerful

24 machine learning method, with eleven predictor variables to forecast the spatial occurrence as

25 well as the abundance distribution. The results show that the occurrence model had a decent

26 performance (ROC: 0.77 ) and the abundance model had a RMSE of 26.54. It is noteworthy that

27 environmental variables influenced bustard occurrence and abundance differently. The area of

28 farmland, and the distance to residential areas were the top important variables influencing

29 bustard occurrence. In contrast, the distance to national roads and to expressways were the most

30 important to variables influencing abundance. In addition, the occurrence and abundance models

31 displayed different spatial distribution patterns. The regions with a high probability of occurrence 
32 were concentrated in the south-central part of the study area; and the abundance distribution

33 showed high populations occurrence in the central and northwestern parts of the study area. Still,

34 combining occurrence and abundance indices to produce a priority protection index (PI) to be

35 used for conservation could guide the protection of the areas with high occurrence and high

36 abundance (e.g., in Strategic Conservation Planning). Due to the widespread use of SDMs and

37 the easy subsequent employment of SAMs, these findings have a wide relevance and

38 applicability. We promote and strongly encourage to further testing, application and updates to

39 the priority protection index (PI) elsewhere to explore the generality of these findings and

40 methods that are readily available now to researchers. 


\section{INTRODUCTION}

42 The knowledge of species occurrence and abundance distribution provides a fundamental

43 information for conservation biology (VanDerWal et al., 2009; Drew et al., 2011; Primack, 2012;

44 Johnston et al., 2015). Understanding how environmental factors are related to species occurrence

45 and abundance distribution and how they are explicit in time and space are priorities in current

46 biodiversity conservation (Drew et al., 2011; Martín et al., 2012).

47 Species distribution models (SDMs) are empirical ecological models that relate species

48 observations to environmental predictors (Guisan \& Zimmermann, 2000); usually this process is

49 done using machine learning algorithms (Drew et al., 2011, see Mi et al., 2017 for an

50 application). SDMs have become important and essential tools in ecology, biogeography, climate

51 change research, conservation, and management because of on their spatial occurrence prediction

52 capacities (Peterson et al., 2002; Guisan \& Thuiller 2005; Elith et al., 2006; Araújo \& New 2007;

53 Mi et al., 2016). SDMs built with count data are called species abundance models (SAMs) (Elith

54 \& Leathwick 2009; Barker et al., 2014; see Yen et al 2004 for an application). SAMs are still less

55 commonly used yet, despite providing valuable information for conservation and management.

56 However, increasing attention has been paid to these problems in recent years (e.g., Yen et al.,

57 2004; Martín et al., 2012; Howard et al., 2015; Ashcroft et al., 2017; Fox et al., 2017).

58 In the past, spatial conservation decisions and plans were usually just based on SDMs (e.g.,

59 Suárez-Seoane et al., 2008; Gray et al., 2009; Adams et al., 2016; Mi et al., 2016). However,

60 despite statements by Newton (2008), many scholars found that species occurrence and

61 abundance distribution did not to display similar patterns (Yen et al., 2004; Karlson et al., 2011; 
62 Yin \& He 2014; Johnston et al., 2015). The difference may represent a mixture of effects and may

63 reflect the differences between the underlying biological processes that of abundance and

64 occurrence (Johnston et al., 2015). Therefore, conservation decisions only based only on SDMs

65 predictions are insufficient and may even be misleading, the same applies for SAMs. In the

66 future, one time-critical challenge and associated progress will be centered how to combine the

67 useful information that SDMs and SAMs each offer for conservation.

68 In this study, we evaluated a case study using the endangered Great bustard (Otis tarda

69 dybowskii), which winters in Cangzhou in the North China Plain near Bohai Bay. This area is one

70 of the most important wintering grounds for this species (approximately 300 individuals,

71 c.13.6 20.0\% of China's total wintering population (Goroshko 2010; Meng 2010)). Using the

72 Great Bustard as a case study would contribute to our conservation knowledge about the habitat

73 use of this threatened species and enable us to design better conservation policies. By studying

74 not only the spatial occurrence and the abundance patterns but also combining these two model

75 types together as a role model, predictive modeling and its inferences would potentially have

76 wider conservation implications. Our overall objective of this research was to (1) assess and

77 develop models to accurately predict the patterns of bustard occurrence and abundance; (2)

78 identify the environmental variables that influence the occurrence and abundance of this species;

79 (3) combine occurrence and abundance models as a new contribution to conservation decisions;

80 and (4) investigate the overall relationship among predicted occurrence, predicted abundance and

81 observed abundance. Well-tested and suitable methods used in this research could be useful for

82 the conservation of the Great Bustard, and other rare species; additionally, this research could

83 generally improve biodiversity through the application of SDMs and SAMs. 


\section{MATERIALS AND METHODS}

85 Study area

86 This study was conducted at the wintering grounds of the endangered Great Bustards in

87 Cangzhou, southeast of the Heibei Province in the wider Bohai Bay (Fig. 1). It is located at $8838^{\circ} 12^{\prime} 57^{\prime \prime}-38^{\circ} 36^{\prime} 51^{\prime \prime}$ latitude and at $116^{\circ} 50^{\prime} 48^{\prime \prime}-117^{\circ} 24^{\prime} 03^{\prime \prime}$ longitude in the warm temperate,

89 semi-humid monsoon climate zone, which features the slightly marine climatic characteristic of

90 the Bohai Sea region. The topographical and climate conditions vary little in the study area

91 (altitude varies by $13 \mathrm{~m}$, temperature by $0.4^{\circ} \mathrm{C}$, and precipitation is the same). The total study 92 area is $2,191.4 \mathrm{~km}^{2}$, consisting of farmland $\left(1,675.1 \mathrm{~km}^{2} ; 76.4 \%\right)$, residential area $\left(330.5 \mathrm{~km}^{2}\right.$, $9315.1 \%)$, open water $\left(23.5 \mathrm{~km}^{2} ; 1.1 \%\right)$ and other unspecified land uses (e.g., home lots, sheds).

95 Most of the farms in this region produce cereal, which is grown in a 2-year rotation system. In

96 the first year, winter cereal is cultivated from early September to the end of April of the following

97 year. Then, corn is cultivated between the end of April to early September of the same year. The

98 study area was chosen (Fig. 1) because of its large number and proportion (approximately 300

99 individuals, c.13.6 20.0\% of China's total wintering Great Bustard population (Goroshko 2010;

100 Meng 2010). This area is the world's largest wintering ground of the endangered O. t. dybowskii.

101 This area is representative of the typical farmland in the North China Plain. In addition, accurate

102 Great Bustard census data, geographic information system (GIS) data coverages and satellite 103 imagery were readily available. 
104 Bird census data

105 Spatial occurrence and abundance data for Great Bustards were used to develop models. A

106 Great Bustard census was conducted between November 2013 and March 2014. In the study area,

107 we travelled with a small four-wheel-drive tractor along the roads between farmland, at speeds

108 between $10-30 \mathrm{~km} /$ hour. We are confident we have an equal and virtually complete detectability

109 in the study area. No great bustard flocks were overlooked. Our team consisted of two

110 experienced observers (one surveyor and one local resident) carrying out the bustard counts, and

111 this team was familiar with the survey area. When a flock was found, we drove slowly and

112 stopped at a location approximately 100 - $500 \mathrm{~m}$ distance from the bustard flocks, then, we

113 recorded the size, location, habitat type and basic behavior of the flock. This resulted in the high

114 detection of birds and flocks in the study area because birds can be seen from long distances

$115(\sim 3 \mathrm{~km})$ and also when flying away. The actual coordinates of the animal locations were obtained

116 by Google Earth by combining it with our recorded locations. Each census conducted from dawn

117 until dusk. During the study, we identified 94 bustard sites within the study area. To our

118 knowledge, this census data comprises the best available data for bustards in China.

119 GIS environmental layers

120 Based on the environmental conditions in our study area, we selected eleven habitat and

121 landscape (i.e., environmental) variables to construct models that predict occurrence and

122 abundance (Table 1). To obtain these variables, we acquired the basemap from Google Earth

123 (using Daogle, an open source software made by a Chinese individual http://www.daogle.com/;

124 as used and explained in Mi et al., 2014) and derived otherwise unavailable high-resolution 
125 landscape inventory information about open-water pools, rivers, residential areas, national roads,

126 provincial roads, expressway, farmland roads, ditches and farmland areas from the base map.

127 Next, we constructed a distance layer for these variables (except for the farmland area) using the

128 Euclidean Distance tool in ArcGIS 10.1 with a $30 \mathrm{~m} \times 30 \mathrm{~m}$ pixel size (ArcToolBox-Spatial

129 Analyst Tools-Distance-Euclidean Distance). This high-pixel resolution was chosen to maintain

130 consistency with the remote sensing variable resolution we used.

131 Satellite images

132 A range of the best cloud-free HJ-1A/B (HuanJing (HJ)) satellite images

133 (http://218.247.138.121/DSSPlatform/index.html\#) with $30 \mathrm{~m} \times 30 \mathrm{~m}$ resolution were obtained for

134 each month between November 2013 and March 2014 in order to calculate the normalized

135 difference vegetation indices (NDVI) signature for each pixel. The HJ-1A/B CCD data were run

136 for radiometric calibration, atmospheric correction and geometric correction to obtain surface

137 reflectance data and subsequent NDVI data. Radiometric calibration was finished using 2014 HJ-

138 1A/B CCD absolute radiometric calibration coefficients, which were provided by the China

139 Center for Resources Satellite Data and Application. For this study, we used maximum and mean

140 NDVI to represent the vegetation conditions (Osborne et al., 2001). 
142 Model development

143 We employed an machine learning technique, Random Forest, to model the occurrence as well

144 as the abundance distribution of Great Bustards. Breiman's (2001) Random Forest

145 implementation in SPM7 by Salford Systems Ltd. is robust to over-fitting and is widely

146 recognized to produce high-quality predictive models (Mi et al., 2017). Hence, Random Forest is

147 increasingly applied to species distribution modelling (Cutler et al., 2007; Drew et al., 2011; Mi

148 et al., 2016 for an application using bustards in China). Though Random Forest performed the

149 best in terms of predicting abundance itself (see Appendix 1), testing the feasibility of other data

150 was essential for maintaining high certainty. Thus, to assess the robustness of the model, we

151 pooled data from 2013 and 2014 , and then used $80 \%$ of the abundance data as training data and

152 the remaining $20 \%$ as testing data. When we constructed initial abundance models with all eleven

153 environmental predictors, model performance was not good (the $\mathrm{R}^{2}$ value was small). This was

154 likely due to the regression settings in the Random Forest algorithm. For a better outcome, we

155 assessed a "stepwise" setting in SPM for all of the abundance data (100\%), re-run the models,

156 and obtained better results. As a result, we identified a multivariate set of four environmental

157 predictors (distance to expressway, distance to national road, distance to pool, and MNNDVI),

158 which had the best performance (the biggest $\mathrm{R}^{2}$ value). Using these four predictors, we

159 reconstructed the abundance model based on the training data (80\%) and validated it with testing

160 data $(20 \%)$. We found that the regression model performance was acceptable but fair $\left(\mathrm{R}^{2}=0.551\right)$

161 when comparing observed abundance with predicted abundance. Thus, we constructed the final

162 abundance model based on the above four selected variables described above and the entire set of

163 observation data. To obtain an abundance index more closely aligned with the observations, we

164 adjusted the prediction abundance according to the linear regression between prediction

165 abundance and observation. First, we constructed a regression formula based on the known 
166 abundance from observation and prediction [observation abundance $=\mathrm{A} \times$ prediction abundance +

167 B]. Then, once A and B were be known, they were applied to calculate the adjustment 168 abundance $=[$ adjustment abundance $=\mathrm{A} \times$ prediction abundance $+\mathrm{B}]$. The regression relation 169 between observation and prediction abundance are shown in Fig. 2.

171 Further, Random Forest was also applied to rank the relative importance of the environmental

172 variables. In SPMv7, partial dependence plots are not directly implemented in Random Forest but

173 can be obtained easily in R or can be mimicked in the TreeNet model as a Random Forest run.

174 Thus, we used TreeNet with bagging settings to create partial dependence plots for each variable 175 included in the occurrence and abundance models.

176 Approximately 10,000 pseudo-absence points were chosen by random sampling across the 177 study areas using the freely available Geospatial Modeling Environment (GME) software 178 (http://www.spatialecology.com/gme/) for distribution models. In SPM7, we set balanced class 179 weights, grew each model to 1,000 classification trees for the occurrence model and 1,000 180 regression trees for the abundance model, and used all other default setting of the software. We 181 extracted the habitat information for presence and pseudo-absence points for Great Bustards from 182 the environmental layers in GME ("isectpntrst" commands), and then we created a model file in 183 SPM7 called a 'grove' that contained the algorithm that quantified the patterns of occurrence; this 184 was used to score all pixels in the study area. We also extracted the habitat information from the 185 same environmental layers for abundance points and then generated a 'grove' file for abundance 186 to score abundance estimates for each pixel in the study area.

187 For spatial occurrence and abundance distribution visualization, we applied the SPM7 grove 188 files to a regular lattice of points (pixels; also attributed to the environmental variables) spaced at 189 30-m intervals across the study area. Model outputs generated relative indices of occurrence 190 (RIO; an index of pixels from 0 to 1 that represent a relative index belonging to the 'occurrence' 
191 class) and a relative abundance index (prediction abundance) for each point in the regular lattice

192 based on its underlying environmental variables. We also adjusted the predicted abundance based

193 on a linear regression as constructed in the previous model development steps (Fig. 2a). For a

194 better continuous spatial visualization, the RIO and predicted abundance values were smoothed

195 between neighboring points across the extent of the study area using the Inverse Distance

196 Weighting (IDW) tool in ArcGIS 10.1. This yielded spatially continuous predictive distribution

197 and abundance raster maps of for the Great Bustard.

198 Model validation

199 The Random Forest performance was first assessed internally using a set of 'out-of-bag' (OOB)

200 training points (OOB; a specific concept used with Random Forest models to describe a subset of

201 points not used initially for model fitting; Breiman 1996, Breiman 2001). Using this out-of-bag

202 dataset, the receiver operating characteristic (ROC) and RMSE were used to calculate the

203 predictive performance of the occurrence and abundance models, respectively (Zweig and

204 Campbell 1993; Fielding and Bell 1997; Huettmann and Gottschalk 2010).

205 Priority protection analysis

206 To have a more suitable and scientific protection plan for the endangered Great Bustard, in this

207 study, we developed and proposed the use of an index called the priority protection index (PI),

208 which combines the predicted results of the SDM and SAM. This index is calculated by the

209 following equation for each site:

$$
P I=\frac{R I O \times R A}{\max (R I O \times R A)}
$$

210 where $\boldsymbol{P I}=$ priority protection index (an index of pixels from 0 to 1 that represent the priority of

211 conservation), $\boldsymbol{R I O}=$ relative index of occurrence, and $\boldsymbol{R} \boldsymbol{A}=$ relative abundance (prediction

212 abundance). In our study, we computed the PI for the entire study area based on the RIO and the

213 adjusted RA value for each grid cell of the spatial occurrence and abundance maps. Then, we 
214 used the IDW tool in ArcGIS 10.1 to generate spatially continuous priority protection index (PI)

215 raster maps. In this equation, we did not consider the weighting the biotic and socioeconomic 216 variables. Therefore, the justification and use of the PI should be explained a little more: when

217 combining the SDM with the SAM, one will not find a straight forward relationship between 218 occurrence and abundance (see Yen et al. 2004 for an example). What the PI will do, but what has 219 not been achieved much before, is to essentially model the relationship between occurrence and 220 abundance, and provide a combined view of the occurrence index and abundance index that is 221 explicit in space and time. Achieving this can help to better prioritize the pixels.

\section{RESULTS}

223 Model performance

224 Our distribution model obtained a decent performance (ROC: 0.77 ) according to Fielding and 225 Bell (1997), and the abundance model had a RMSE of 26.54 (RMSE is unit-less).

226 Variable importance

227 Table 2 presents the variable importance ranking for the occurrence and abundance models 228 obtained from the Random Forest method. We found that the area of farmland, the distance to 229 residential areas (buildings), to ditches and to expressways were the top four most important 230 variables that influenced bustard occurrence. Those come as a multivariate ecological package (a 231 combination of many predictors). The NDVI, which represents vegetation conditions, was less 232 important than the other nine predictors. For the abundance model, the most important factors 233 were the distance to national roads and to expressways, followed by water factors (the distance to 234 pools) and food-related factors (MNNDVI) 
236 Partial dependence plots

237 Partial dependence plots could interpret the functional relationships and effects of each variable 238 by representing a variable's marginal effects on the response (Elith et al., 2008; Johnstone et al., 239 2010). It helps to find the signal in the data; Fig. 3a indicates that the occurrence preference of 240 bustards for farmland areas was between 0.6 and $7.5 \mathrm{~km}^{2}$. Additionally, based on model results, it 241 appeared the bustard preference were as follows: distance to residential areas ranged from 250 to 242 2,500 m (Fig. 3b), distance to ditches ranged from 100 to 4,500 m (Fig. 3c), and distance to 243 expressways ranged from 6,000 to 19,000 m (Fig. 3d). In contrast, for abundances, more 244 individuals occurred beyond 2,300 m, but were less than 9,500 $\mathrm{m}$ away from national roads (Fig. $2453 \mathrm{e}$ ); additionally, bustards were found in a range between 7,000 and 11,000 m away from 246 expressways (Fig. 3f). Moreover, this species stayed away from pools (maintaining distance 247 greater than 1,500 m, Fig. 3g) and preferred areas with more vegetation (mean NDVI during the 248 investigation was larger than 0.13, Fig. 3h). The information for other variables, which were 249 more marginal, can be found in Appendix 2.

251 Occurrence, abundance distribution patterns and priority protection

252 Fig. 4 shows the maps of the RIO (relative index of occurrence), adjusted RA (relative 253 abundance) and PI (priority protection index). From the RIO map (Fig. 4a), we found that the 254 distribution area of high RIO for bustards was high. The regions of high possibility of bustard 255 occurrence were concentrated in the south-central study area; and the whole habitats represented 256 a fragmented distribution. The abundance distribution had a different pattern and showed high 257 populations occurrence in the central and northwestern parts of the study area (Fig. 4b). Based on 258 the occurrence and abundance distribution results, we used Equation (1) and obtained the results 259 shown in Fig. 4c. These results indicate that a high PI is located in the center, north and northeast 
260 regions of the study area, and they indicate a sporadic and fragmented distribution, which could

261 represent a priority protection site if a conservation decision was to be made.

262

Put Fig. 4 Here

\section{DISCUSSION}

264 The occurrence and abundance models of Great Bustard developed here were designed to identify 265 relevant locations for where to prioritize conservation, and to assess the effects of each variable 266 that influenced this species' occurrence and abundance (Fig. 3). From a multivariate perspective, 267 the area of farmland, distance to residential areas, distance to ditches and to expressways were 268 among the top four most important predictors for bustard occurrence; in contrast, for the 269 abundance model, the variables consisted of another multivariate package that include the 270 distance to national roads, the distance to expressways, the distance to pools and the mean NDVI

271 (Table 2). We found that high RIO habitats had a fragmented distribution throughout the entire 272 study area (Fig. 4a). The abundance model showed that large populations usually occurred in the 273 central and northwestern parts of our study area (Fig. 4b). The center, north and northeast

274 portions of the study area had a high priority protection index (PI) and had a severely fragmented

275 distribution, including these areas should be the priority sites for protection (Fig. 4c). This not

276 only confirms our own records and, with the help of the PI, can now be quantified and modeled 277 further for more effective conservation.

278 In our study area, human disturbance was very strong and represented by indicators, such as 279 density of roads and residential areas (Fig. 1). During our study, we also found other threats to 280 this endangered species: these included farmers grazing their sheep; famers sprinkling poison 281 baits in the wheat fields to present sheep from entering; some bird photographers pursued 282 bustards by walking or following birds while on motor vehicles to take photos, which they 283 wanted to show off to others; hunters with dogs chasing hare and ring-necked pheasant during the 
284 day and night; some local people hunting bustards; increasing power lines construction in 285 agricultural lands, resulting in bustards sometimes colliding with wires, getting injured, or even 286 dying, especially flying on foggy days or when in a hurry (Janss \& Ferrer 2000); and the 287 interference of firecracker sounds during the Chinese Spring Festival as well as oil rigs and wind 288 farms. Though carrying a high disturbance can result in stress synthesis (e.g., "death by thousand 289 cuts"), a large number of wintering bustards (approximately 300, c. $13.6 \sim 20.0 \%$ of China's total 290 wintering population; Goroshko 2010; Meng 2010) still wintered in this area. In times of climate 291 change, it can be assumed the population widens (Mi et al., 2016). Thus, this is an area of 292 essential importance for bustards in China, regardless of which perspective is taken. Therefore, a 293 feasible conservation plan should be designed, and based on our model's prediction result, 294 combined with local public customs and financial support as well as a wider buy-in. In our 295 opinion, improving the local pupulation's education on animal protection, as has been done over 296 the years, would be useful. The same applies to increasing budgets, enforcement and frequency of 297 patrol by the local management and conservation NGOs in the regions with high PI value, and the 298 local community and government should provide more financial support. However, when 299 designing patrol routes designation in the field, the individuals who are monitoring should avoid 300 getting too close to bustards, so as not to disturb and stress the regular wintering activities of the 301 bustards. For the benefit of this species and its habitats, we suggest not converting crop farmland 302 into nursery farmland; and we encourage farmers to harvest their crops with a machine, which is 303 a more beneficial harvesting method for bustards based on our previous research results (Mi et 304 al., 2014). We also highly recommend, if possible, to bury power lines into the ground and to 305 collect hunting guns from the local public.

306 In this study, occurrence and abundance did not display identical spatial distribution patterns, a 307 result which has been reported in some previous studies (Conlisk et al., 2007; Karlson et al., 308 2011; Yin \& He 2014; Johnston et al., 2015). There is actually no reason to assume a presence 
309 site represents only one individual animal, nor should a linear relationship between RIO and

310 abundance be assumed. Technically speaking, 'presence' can mean one to infinite animals are

311 present, and details depend on the actual pixel setup and how it fits into the obtained model.

312 Therefore, while the relationship is not automatically clear, this could be due to several reasons

313 and depending on specific habitat details: Firstly, the environmental variables that contributed to

314 occurrence and abundance were different, as indicated in Table 2. Secondly, the predictors of

315 preference for bustard occurrence and abundance models were different. For instance, bustards

316 occurred in areas with a distance to expressways ranging from 6,000 to 19, $000 \mathrm{~m}$ (Fig. 3d), while

317 most populations occurred between 7,000 and 11,000 $\mathrm{m}$ from expressways in terms of abundance

318 (Fig. 3f) (see more details in Fig. 3 and Appendix 2). Thirdly, they differed in their spatial

319 distribution for occurrence and abundance (Fig. 4a, b). Based on the analysis of overlaying the 320 observation sites with the RIO and observation abundance (Fig. 5a, b), the estimated relative 321 index of occurrence (RIO) was not consistently related with the relative index of abundance (Fig.

322 5a). All locations of observed abundance had high RIO (Fig. 5a), and the relationships between

323 occurrence and abundance estimates were nonlinear (Fig. 5b). These differences may represent a

324 mixture of effects that reflect differences between the underlying biological processes that give

325 rise to specific abundance and occurrence at a specific pixel, as well as limitations imposed by

326 the data and methodology used to estimate these patterns (Johnston et al., 2015; see Buckland et

327 al., 2016 for Distance Sampling and detection problems). In addition, how to interpret the

328 inconsistency between these two indices of plant prediction is a problem waiting to be further

329 resolved e.g., between crop occurrence index (equal to habitat suitability index) and crop 330 abundance (e.g., production).

332 When treating all presences reports as equal in species distribution models (SDMs; occurrence 333 model, habitat niche model)-regardless of the abundance of individuals that the habitat supports - 
334 this could provide us with information on the loss of suitable of habitat (Howard et al., 2014).

335 Applying models based on abundance data, even at a relatively coarse scale, can help to predict 336 spatial patterns of occurrence that are modelled with even greater refinement (Howard et al., 337 2014). Conservation decision-making should use as much knowledge and information as possible 338 to optimize the benefits of conservation actions (Sutherland et al., 2004; Segan et al., 2011). The 339 use of species distribution models (SDMs) of occurrence has been an important tool in 340 optimizing the selection of protected areas (Franklin 2013; Guisan et al., 2013, Mi et al., 2016; 341 Han et al., 2017) based on the ecological niche space (Drew et al., 2011), but relative abundance 342 is often perceived to be a more relevant metric because it can quantify animals within a pixel, and 343 thus, populations (Johnston et al., 2015). Modeling abundance requires methods that can handle 344 large numbers of zero counts as well as the rare, but important, high counts (Welsh et al., 1996), 345 even without a solid research design, according to frequentist statistics. However, Yen et al., 346 (2004), Magness et al., (2008) and Fox et al., (2017) have already showed how machine learning 347 can change this perspective and provide very powerful solutions.

348 High counts and their locations are particularly important because the pixels with the highest 349 densities of animals are potentially of the greatest interest for conservation planning (Johnston et 350 al., 2015). In our study, we found that the regressions in Random Forest performed poorly at sites 351 with low and high counts (Fig. 2b), although it showed a highly linear relationship between 352 observed and predicted abundance $\left(\mathrm{R}^{2}=0.844\right.$; Fig. $\left.2 \mathrm{a}\right)$. Therefore, we argue that the regression 353 method in the Random Forest algorithm should optimize low- and high-count predictions. We 354 recommend to classifying abundances into bins (e.g., high, medium, and low with associated 355 abundance estimates) because Random Forest is exceptionally strong for classification problems. 356 However, for now, this remains an open field of research, but we find our progress remains 357 substantial.

358 Abundance data could also provide valuable baselines against which to assess future changes 
359 (Cumming 2007) (e.g., climate change, land use change). Such changes in abundance will be

360 much more rapidly apparent, and hence, more rapidly detected than changes in presence-absence

361 patterns across large ranges (Gregory et al., 2005). However, only a few spatial distribution

362 modelers derived models based on the collection of abundance data (e.g., Yen et al. 2004, Fox et

363 al. 2017). This may be because collection of abundance data is more cost or resource demanding

364 than collecting presence - absence data especially for highly mobile animals. Such data are

365 sophisticated in structure and research design, and still they are rarely shared (see in GBIF.org).

366 Therefore, we recommend that abundance data could be collected (and can also easily to be

367 turned into presence-absence data, too), even at only relatively coarse numerical scales, because

368 the benefits are considerable (as stated by Howard et al., 2014). One thing that should be

369 mentioned is that plenty of abundance data and models did not perform well and, abundance was

370 extremely difficult to predict (Oppel et al., 2012). Finding the underlying causes that influence

371 abundance model accuracy and constructing more accurate models would be extremely important

372 and useful in future applications toward individual-based policy applications.

373 For a spatial priority protection of mobile species, one should note that high numbers of

374 individuals are not always present in the same habitats and pixels; instead, low numbers of

375 individuals may occur in one place many times. In addition, this may have implications on spatial

376 priority protection for mobile species. Previous studies have used analytical approaches to

377 address some of these challenges (e.g., Nichols et al., 2009; Kery \& Andrew Royle 2010; Oppel

378 et al., 2012; Jiguet et al., 2013). However, no general modeling framework has been proposed for

379 dealing with all these analytical challenges simultaneously. This is exactly where our PI offers

380 progress. We also thought the situation of mobile species selecting habitats could be divided into

381 five scenarios: higher numbers and multi frequency, higher numbers and lower frequency, low

382 numbers and multi frequency, low numbers and low frequency, and none. When a conservation

383 plan is designed for a species, one should consider not only occurrence index and frequency but 
384 also abundance. Here, we proposed the priority protection index (PI; Equation (1) and Fig. 4),

385 based on the distribution of occurrence and abundance patterns, as a helpful tool for more quickly

386 designs a priority protection plan compared to indices, and it is only based on distribution of

387 occurrence or abundance.

388 To date, quantitative estimates of population size during global and local changes have actually 389 proven to be difficult to forecast (O'Grady et al., 2004). This is a major hindrance for effective 390 management, as population size and trends are considered among the best correlates of extinction 391 risk (O'Grady et al., 2004). Such measures are commonly used in determining the conservation 392 status of a species (e.g., IUCN (2001)). We argue that habitat loss remains the one and only 393 powerful metric that can be obtained quickly on a landscape-scale in the absence of proper trend 394 and abundance data (e.g., Drew et al. 2011). The relationship between predicted environmental 395 suitability and abundance - as presented here - may provide us with a possible method for 396 predicting population size and its changes associated with distributional changes; additionally, it 397 may be particularly appropriate for non-mobile species (e.g., plants, fungi). However, this method 398 is not particularly suitable for mobile species, especially for highly mobile species, such as many 399 birds, bats, and flying insects. They may move over a large landscape within a single day, and 400 abundance and the environment conditions can vary seasonally and spatially. When computing 401 population size or population density using abundance, the primary task will be determining the 402 appropriate unit area for investigation and conservation management.

403 This study is the first that has combined model-predicted occurrence (representing species 404 distribution models) and abundance indices (representing species abundance models) to produce 405 a priority protection index (PI), which may contribute to spatial conservation and management 406 decisions worldwide. We strongly encourage other researchers to test, apply and update the 407 priority protection index (PI) to explore the generality of these findings further. 


\section{Acknowledgements}

409 We thank Min Liu for his hard work in the field, Jianguo Fu's great bustard photograph. Thanks

410 also to a shared field survey among the authors. Further thanks go to Salford Systems Ltd. for 411 providing the SPM software. 


\section{REFERENCES}

413 Adams MP, Saunders MI, Maxwell PS, Tuazon D, Roelfsema CM, Callaghan DP, Leon J,

414 Grinham AR, and O'Brien KR. 2016. Prioritizing localized management actions for seagrass

415 conservation and restoration using a species distribution model. Aquatic Conservation

$416 \quad$ Marine and Freshwater Ecosystems 26:639-659.

417 Araújo MB, New M. 2007. Ensemble forecasting of species distributions. Trends in Ecology and 418 Evolution 22:42-47.

419 Ashcroft MB, King DH, Raymond B, Turnbull JD, Wasley J, and Robinson SA. 2017. Moving

420 beyond presence and absence when examining changes in species distributions. Global

$421 \quad$ Change Biology 23:2929-2940.

422 Barker NKS, Cumming SG, Darveau M. 2014. Models to predict the distribution and abundance 423 of breeding ducks in Canada. Avian Conservation and Ecology 9:7.

424 Breiman L. 1996. Bagging predictors. Machine learning 24:123-140.

425 Breiman L. 2001. Random forests. Machine learning 45:5-32.

426 Buckland ST, Rexstad EA, Marques TA, and Oedekoven CS. 2016. Distance Sampling: Methods 427 and Applications. Methods in Statistical Ecology 63:152-153.

428 Conlisk E, Conlisk J, Harte J. 2007. The impossibility of estimating a negative binomial 429 clustering parameter from presence-absence data: a comment on He and Gaston. The $430 \quad$ American Naturalist 170:651-654.

431 Cumming GS. 2007. Global biodiversity scenarios and landscape ecology. Landscape Ecology $432 \quad 22: 671-685$.

433 Cutler DR, Edwards JrTC, Beard KH, Cutler A, Hess KT, Gibson J, Lawler, JJ. 2007. Random 434 forests for classification in ecology. Ecology 88:2783-2792.

435 Drew CA, Wiersma Y, Huettmann F. 2011. Predictive species and habitat modeling in landscape 
436 ecology: concepts and applications. Springer, London.

437 Elith J, Graham CH, Anderson RP, Dudík M, Ferrier S, Guisan A, Hijmans RJ, Huettmann F, 438 Leathwick JR, Lehmann A, Li J, Lohmann LG, Loiselle BA, Manion G, Moritz C, 439 Nakamura M, Nakazawa Y, Overton JMM, Peterson AT, Phillips SJ, Richardson K, 440 Scachetti-Pereira R, Schapire RE, Soberón J, Williams S, Wisz MS, and Zimmermann NE. 441 2006. Novel methods improve prediction of species' distributions from occurrence data. $442 \quad$ Ecography 29:129-151.

443 Elith J, Leathwick JR, Hastie T. 2008. A working guide to boosted regression trees. Journal of $444 \quad$ Animal Ecology 77: 802-813.

445 Elith J, Leathwick JR. 2009. Species distribution models: ecological explanation and prediction 446 across space and time. Annual Review of Ecology, Evolution, and Systematics 40:677-697.

447 Fielding AH, Bell JF. 1997. A review of methods for the assessment of prediction errors in 448 conservation presence/absence models. Environmental conservation 24:38-49.

449 Fox CH, Huettmann F, Harvey GKA, Morgan KH, Robinson J, Williams R, Paquet PC. 2017. 450 Predictions from machine learning ensembles: marine bird distribution and density on 451 Canada's Pacific coast. Marine Ecology Progress Series 566:199-216.

452 Franklin J. 2013. Species distribution models in conservation biogeography: developments and 453 challenges. Diversity and Distributions 19:1217-1223.

454 Goroshko OA. 2010. Present status of population of Great Bustard (Otis tarda dybowskii) in 455 Dauria and other breeding grounds in Russia and Mongolia: distribution, number and 456 dynamics of population, threats, conservation. First International Symposium on 457 Conservation of Great Bustard, Beijing (in Chinese).

458 Gray TN, Borey R, Hout SK, Chamnan H, Collar NJ, and Dolman PM. 2009. Generality of 459 models that predict the distribution of species: conservation activity and reduction of model 460 transferability for a threatened bustard. Conservation biology 23:433-439. 
461 Gregory RD, Strien AV, Vorisek P, Meyling AWG, Noble DG, Foppen RP, Gibbons DW. 2005.

462 Developing indicators for European birds. Philosophical Transactions of the Royal Society

463 of London B: Biological Sciences 360:269-288.

464 Guisan A, Thuiller W. 2005. Predicting species distribution: offering more than simple habitat 465 models. Ecology Letters 8:993-1009.

466 Guisan A, Tingley R, Baumgartner JB, Naujokaitis-Lewis I, Sutcliffe PR, Tulloch AIT, Regan TJ, 467 Brotons L, Mcdonald-Madden E, Mantyka-Pringle C. 2013. Predicting species distributions 468 for conservation decisions. Ecology Letters 16:1424-1435.

469 Guisan A, Zimmermann NE. 2000. Predictive habitat distribution models in ecology. Ecological $470 \quad$ Modelling 135:147-186.

471 Han X, Guo Y, Mi C, Huettmann F, and Wen L. 2017. Machine Learning Model Analysis of 472 Breeding Habitats for the Black-necked Crane in Central Asian Uplands under 473 Anthropogenic Pressures. Scientific Reports 7:6114.

474 Howard C, Stephens PA, Pearce-Higgins JW, Gregory RD, Willis SG. 2014. Improving species 475 distribution models: the value of data on abundance. Methods in Ecology and Evolution $476 \quad 5: 506-513$.

477 Howard C, Stephens PA, Pearce-Higgins JW, Gregory RD, Willis SG. 2015. The drivers of avian 478 abundance: patterns in the relative importance of climate and land use. Global Ecology and $479 \quad$ Biogeography 24:1249-1260

480 Huettmann F, Gottschalk T. 2010. Simplicity, Model Fit, Complexity and Uncertainty in Spatial 481 Prediction Models Applied Over Time: We Are Quite Sure, Aren’t We? Springer New York. 482 IUCN. 2001. UCN red list categories and criteria: version 31 Prepared by the IUCN Species 483 Survival Commission.

484 Janss GFE, Ferrer M. 2000. Common Crane and Great Bustard Collision with Power Lines: 485 Collision Rate and Risk Exposure. Wildlife Society Bulletin 28:675-680. 
486 Jiguet F, Thomas C, Cook A, Newson S, Ockendon N, Rehsch M, Roos S, Thaxter C, Brown A,

487 Crick H. 2013. Observed and predicted effects of climate change on species abundance in 488 protected areas. Nature Climate Change 3:1055-1061.

489 Johnston A, Fink D, Reynolds MD, Hochachka WM, Sullivan BL, Bruns NE, Hallstein E, 490 Merrifield MS, Matsumoto S, Kelling S. 2015. Abundance models improve spatial and 491 temporal prioritization of conservation resources. Ecological Applications 25:1749-1756.

492 Johnstone JF, Hollingsworth TN, Chapin FS, Mack MC. 2010. Changes in fire regime break the 493 legacy lock on successional trajectories in Alaskan boreal forest. Global Change Biology $494 \quad 16: 1281-1295$.

495 Karlson RH, Connolly,SR, Hughes TP. 2011. Spatial variance in abundance and occupancy of $496 \quad$ corals across broad geographic scales. Ecology 92:1282-1291.

497 Kery M, Andrew RJ. 2010. Hierarchical modelling and estimation of abundance and population 498 trends in metapopulation designs. Journal of Animal Ecology 79:453-461.

499 Magness DR, Huettmann F, Morton JM. 2008. Using Random Forests to provide predicted 500 species distribution maps as a metric for ecological inventory and monitoring programs. pp 501 209-229 In Smolinski TG, Milanova MG, Hassanien AE Applications of Computational 502 Intelligence in Biology: Current Trends and Open Problems Studies in Computational 503 Intelligence, Vol 122, Springer-Verlag Berlin, Heidelberg.

504 Martín B, Alonso JC, Martín CA, Palacín C, Magaña M, Alonso J. 2012. Influence of spatial 505 heterogeneity and temporal variability in habitat selection: A case study on a great bustard 506 metapopulation. Ecological Modelling 228:39-48.

507 Meng D. 2010. Study on the Rescue to Great Bustard in Cangzhou, Hebei. First International 508 Symposium on Conservation of Great Bustard, Beijing (in Chinese).

509 Mi C, Huettmann F, Guo Y. 2014. Obtaining the best possible predictions of habitat selection for 510 wintering Great Bustards in Cangzhou, Hebei Province with rapid machine learning 
511 analysis. Chinese Science Bulletin 59:4323-4331.

512 Mi C, Huettmann F, Guo Y. 2016. Climate envelope predictions indicate an enlarged suitable 513 wintering distribution for Great Bustards (Otis tarda dybowskii) in China for the 21st $514 \quad$ century. PeerJ 4:e1630.

515 Mi C, Huettman F, Guo Y, Han X, Wen L. 2017. Why choose Random Forest to predict rare 516 species distribution with few samples in large undersampled areas? Three Asian crane 517 species models provide supporting evidence. PeerJ 5:e2849.

518 Newton I. (2008) Migration Ecology of Birds. London, UK: Academic Press.

519 Nichols JD, Thomas L, Conn PB. 2009. Inferences about landbird abundance from count data: 520 recent advances and future directions pp 201-235 In Thomson DL, Cooch EG, Conroy MJ 521 Modeling demographic processes in marked populations Springer, US.

522 O'Grady JJ, Reed DH, Brook BW, Frankham R. 2004. What are the best correlates of predicted 523 extinction risk? Biological Conservation 118:513-520.

524 Oppel S, Meirinho A, Ramírez I, Gardner B, O’Connell AF, Miller PI, Louzao M. 2012. 525 Comparison of five modelling techniques to predict the spatial distribution and abundance of 526 seabirds. Biological Conservation 156:94-104.

527 Osborne PE, Alonso J, Bryant R. 2001. Modelling landscape-scale habitat use using GIS and 528 remote sensing: a case study with great bustards. Journal of Applied Ecology 38:458-471.

529 Peterson AT, Ortega-Huerta MA, Bartley J, Sánchez-Cordero V, Soberón J, Buddemeier RH, 530 Stockwell DR 2002. Future projections for Mexican faunas under global climate change 531 scenarios. Nature 416:626-629.

532 Primack RB. 2012. A primer of conservation biology. 5th Edition Sunderland, Massachusetts. 533 Sinauer Associates.

534 Segan DB, Bottrill MC, Baxter PWJ, Possingham HP. 2011. Using conservation evidence to 535 guide management. Conservation Biology 25:200-202. 
536 Suárez-Seoane S, Morena ELGDL, Prieto MBM, Osborne PE, and Juana ED. 2008. Maximum 537 entropy niche-based modelling of seasonal changes in little bustard ( Tetrax tetrax ) 538 distribution. Ecological Modelling 219:17-29.

539 Sutherland WJ, Pullin AS, Dolman PM, Knight TM. 2004. The need for evidence-based 540 conservation. Trends in Ecology and Evolution 19:305-308.

541 VanDerWal J, Shoo LP, Johnson CN, Williams SE. 2009. Abundance and the environmental 542 niche: environmental suitability estimated from niche models predicts the upper limit of 543 local abundance. The American Naturalist 174:282-291.

544 Welsh AH, Cunningham RB, Donnelly CF, Lindenmayer DB, 1996. Modelling the abundance of 545 rare species: statistical models for counts with extra zeros. Ecological Modelling 88:297546308.

547 Yen PPW, Huettmann F, Cooke F. 2004. A large-scale model for the at-sea distribution and 548 abundance of Marbled Murrelets (Brachyramphus marmoratus) during the breeding season 549 in coastal British Columbia, Canada. Ecological Modelling 171:395-413.

550 Yin D, He F. 2014. A simple method for estimating species abundance from occurrence maps. $551 \quad$ Methods in Ecology and Evolution 5:336-343.

552 Zweig MH, Campbell G, 1993. Receiver-operating characteristic (ROC) plots: a fundamental 553 evaluation tool in clinical medicine. Clinical Chemistry 39:561-577. 


\section{Table $\mathbf{1}$ (on next page)}

Comparison of features around 94 sites occupied by great bustards and 10000 random points.

Values are means \pm standard deviations. 
Table 1 Comparison of features around 94 sites occupied by great bustards and 10000 random points. Values are means \pm standard deviations.

\begin{tabular}{lllcc}
\hline Layer & Variable & Description & Bustard sites & Random points \\
\hline 1 & Distance to pool & Distance to pool in meter & $1179.0 \pm 734.5$ & $1378.0 \pm 910.3$ \\
2 & Distance to river & Distance to river in meter & $2302.0 \pm 1751.2$ & $2630.0 \pm 2483.0$ \\
3 & Distance to residential & Distance to residential in meter & $935.0 \pm 586.8$ & $980.2 \pm 723.8$ \\
4 & Distance to national road & Distance to national road in meter & $5280.0 \pm 4234.2$ & $5855.0 \pm 4036.9$ \\
5 & Distance to provincial road & Distance to provincial road in meter & $8730.0 \pm 5928.7$ & $9217.0 \pm 6112.4$ \\
6 & Distance to expressway & Distance to expressway in meter & $10010 \pm 5750.0$ & $9585.0 \pm 6666.7$ \\
7 & Distance to farmland road & Distance to farmland road in meter & $477.4 \pm 385.3$ & $524.9 \pm 455.8$ \\
8 & Distance to ditch & Distance to ditch in meter & $1522.0 \pm 1722.7$ & $2120.0 \pm 2078.1$ \\
9 & Area of farmland & Area of farmland in kilometers & $3.3 \pm 3.2$ & $5.3 \pm 6.2$ \\
10 & MNNDVI & The average value of the normalized difference vegetation & $0.14 \pm 0.04$ & $0.13 \pm 0.05$ \\
& & & index from November, 2013 to March, 2014 & $0.23 \pm 0.06$ \\
& & The maximum value of the normalized difference vegetation & & $0.21 \pm 0.07$ \\
\end{tabular}


Table 2 (on next page)

Variables importance ranking of occurrence and abundance models. 
Table 2 Variables importance ranking of occurrence and abundance models

\begin{tabular}{ccc}
\hline Ranking & Occurrence model & Abundance model \\
\hline 1 & Area of farmland & Distance to national road \\
2 & Distance to residential & Distance to expressway \\
3 & Distance to ditch & Distance to pool \\
4 & Distance to expressway & MNNDVI \\
5 & Distance to pool & -- \\
6 & Distance to river & -- \\
7 & Distance to provincial road & -- \\
8 & Distance to national road & -- \\
9 & Distance to farmland road & -- \\
10 & MAXNDVI & -- \\
11 & MNNDVI & -- \\
\hline
\end{tabular}


Figure 1

Study area and bird abundance and occurrence data for Great Bustard in Cangzhou, China.

Photograph of Great Bustard by Jianguo Fu.

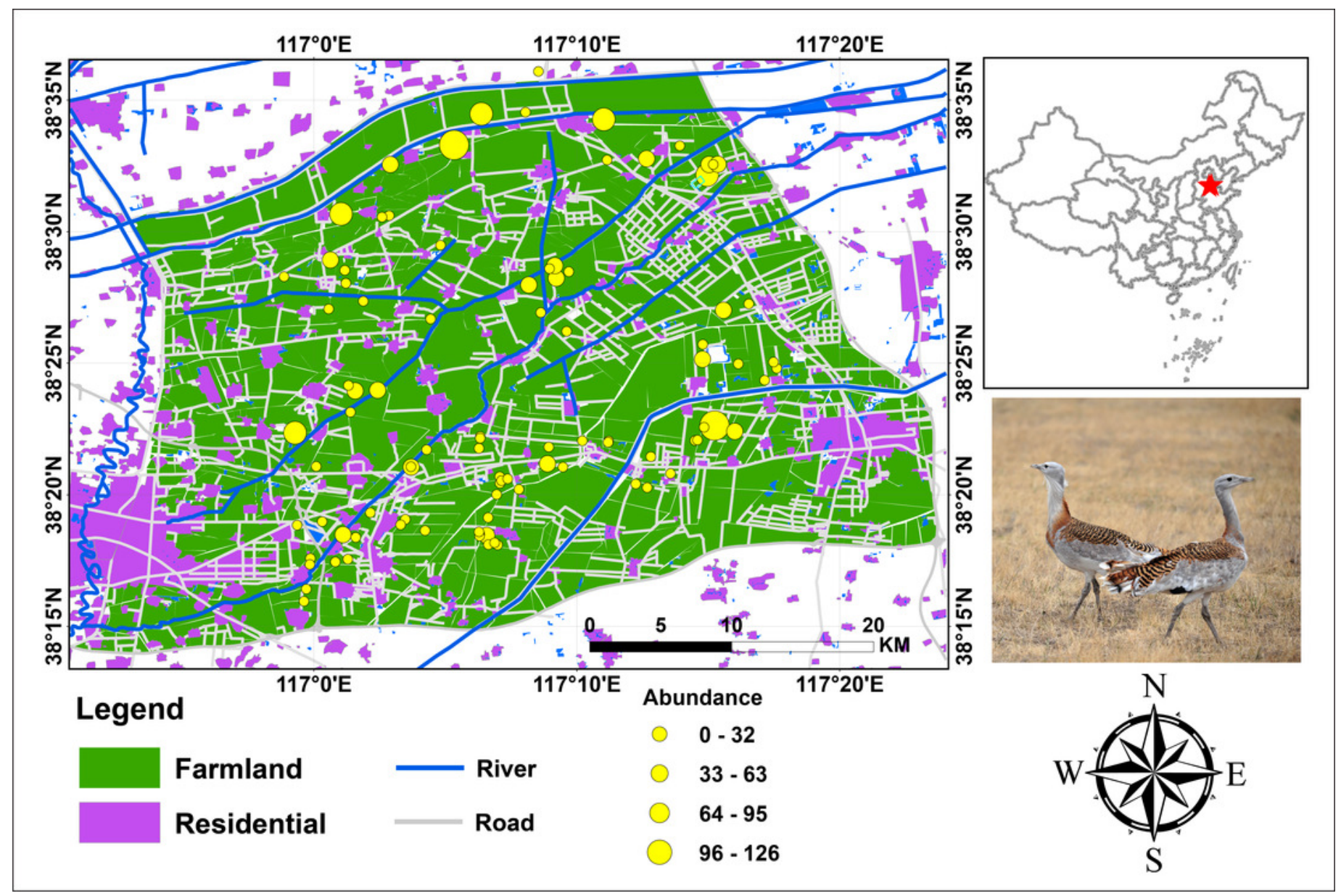




\section{Figure 2 (on next page)}

Figure 2

The relationship between observation and prediction abundance using Random Forest for Great Bustards. (a) Scatter plot of observation abundance with prediction and adjustment prediction abundance, and (b) lines and points plot of observation, prediction and adjustment prediction abundance. 
PeerJ

옴

- Prediction adjustment

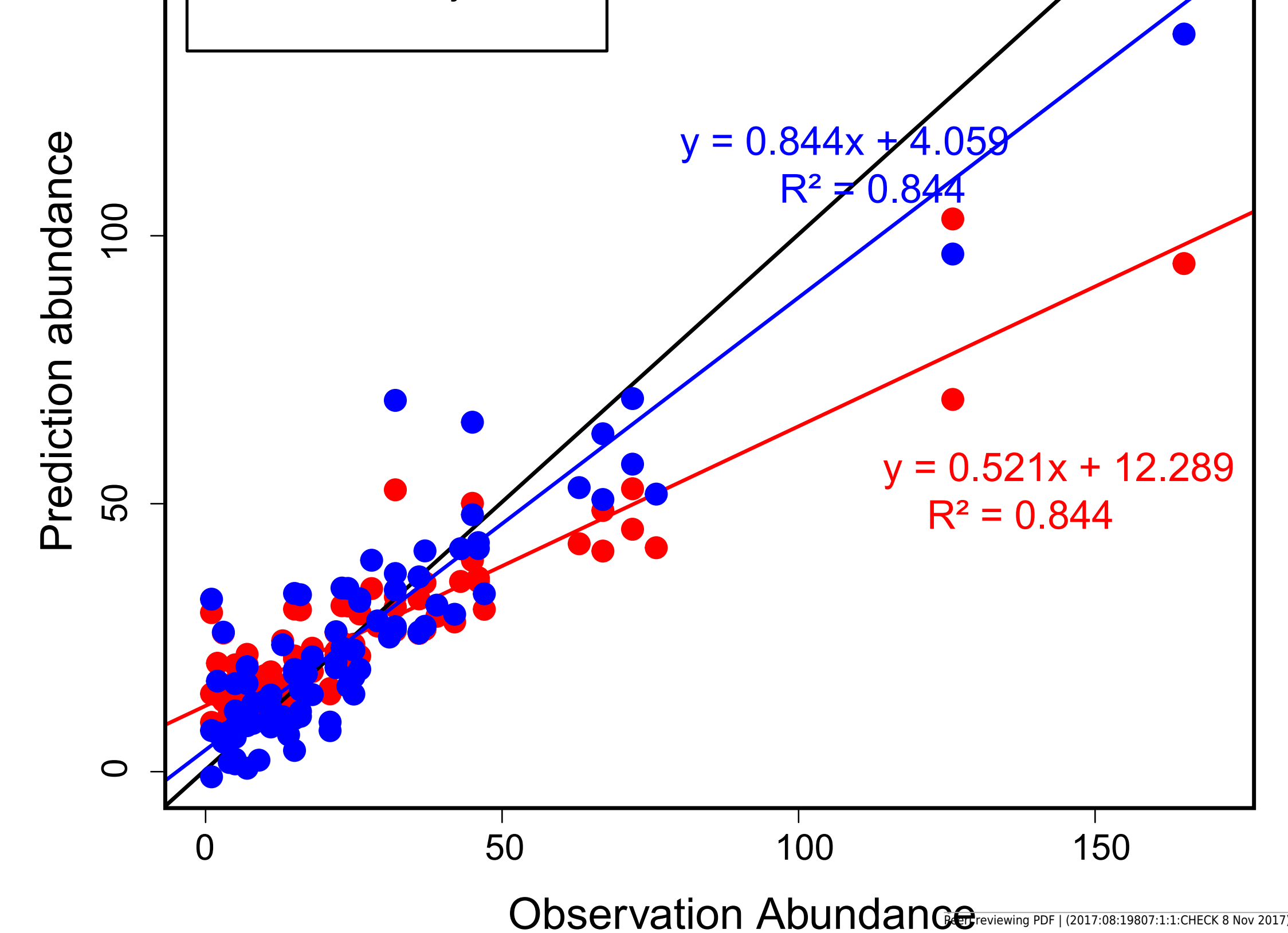

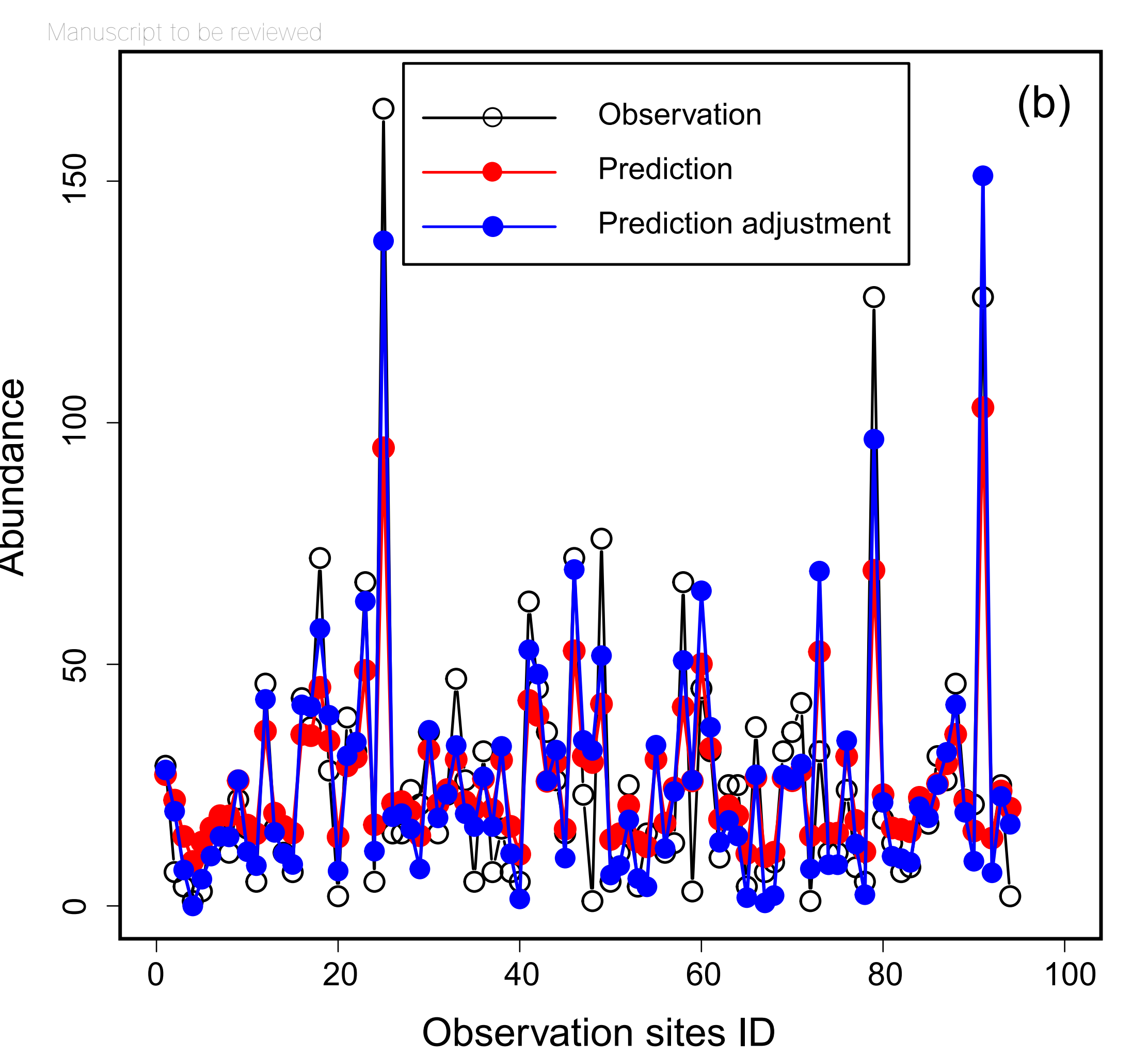




\section{Figure 3 (on next page)}

Partial dependence plots for the top four most influential variables in the occurrence and abundance distribution models for Great Bustards, respectively.

(a) area of farmland in occurrence distribution model; (b) distance to residential in occurrence distribution model; (c) distance to ditch in occurrence distribution model; (d) distance to expressway in occurrence distribution model; (e) distance to national road in abundance distribution model; (f) distance to expressway in abundance distribution model; (g) distance to pool in abundance distribution model; and (h) mean NDVI in abundance distribution model. 

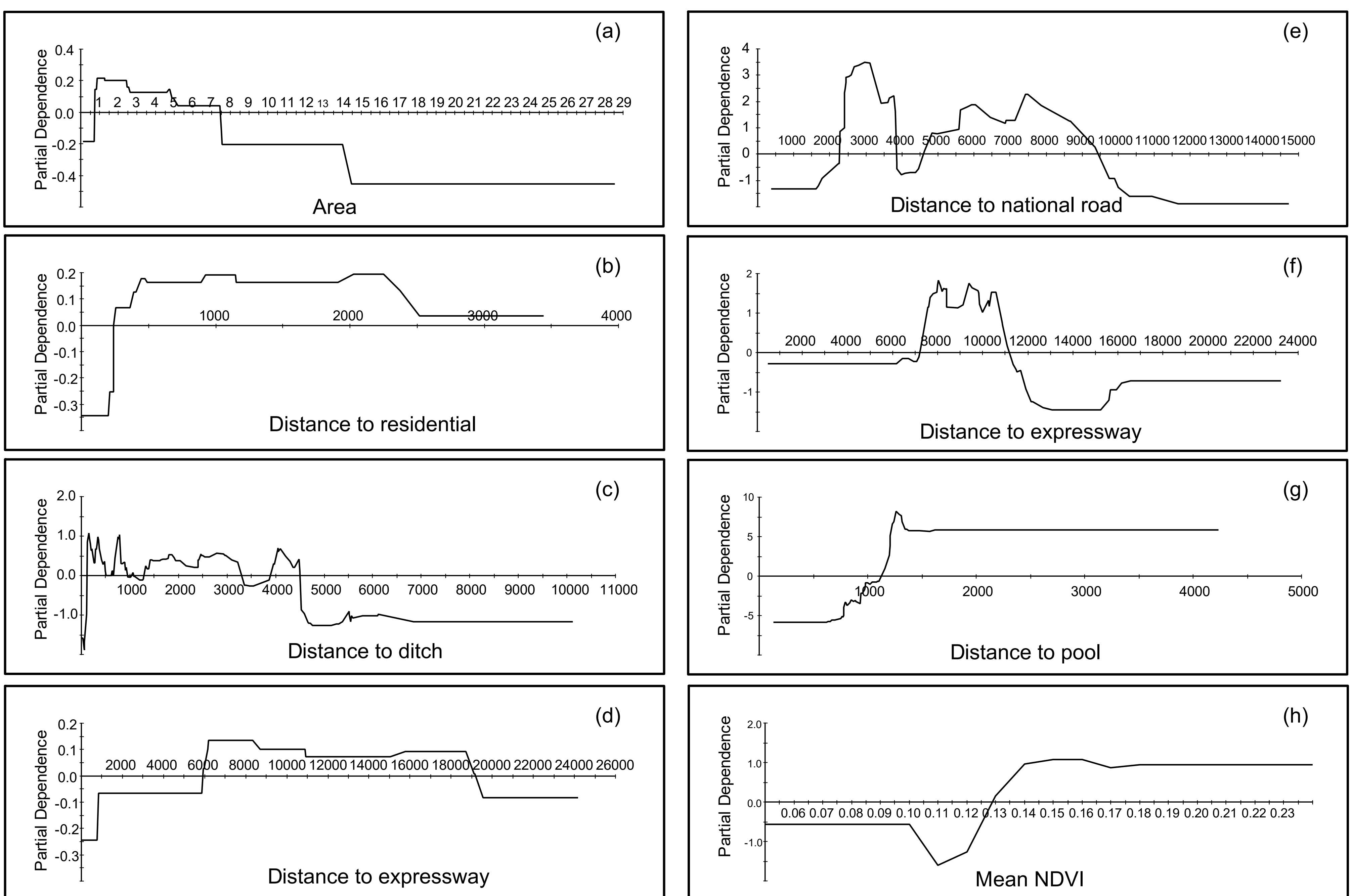


\section{Figure 4}

Spatial distribution map of relative index of occurrence (RIO), relative abundance (RA) and priority protection index (PI).

(a) Map of relative index of occurrence (RIO); (b) map of adjusted relative abundance (RA); and (c) map of priority protection index (PI). 


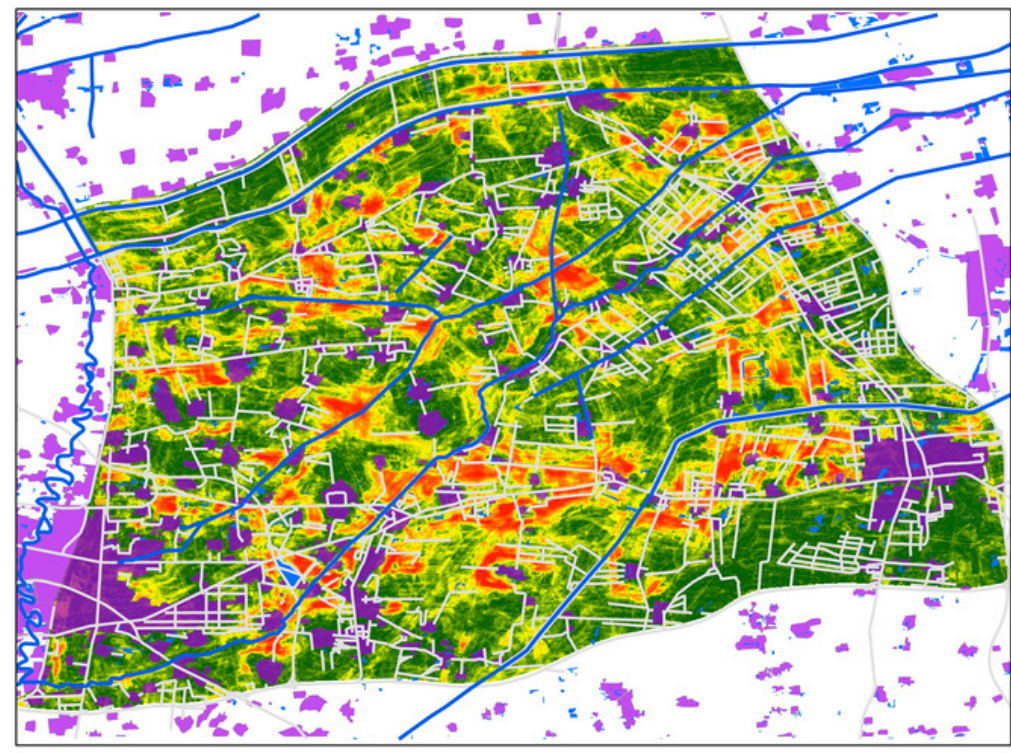

(a)

Relative Index of Occurrence (RIO)

High : 1.00

Low : 0.00

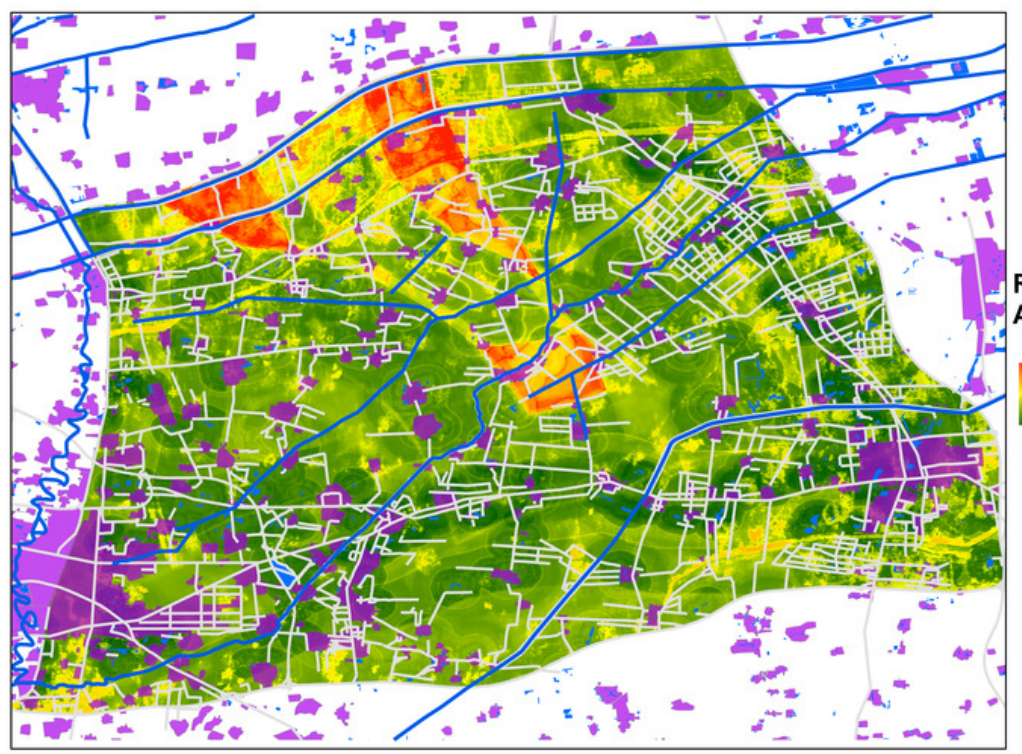

(b)

Relative

Abundance (RA)

High : 152

Low : 0

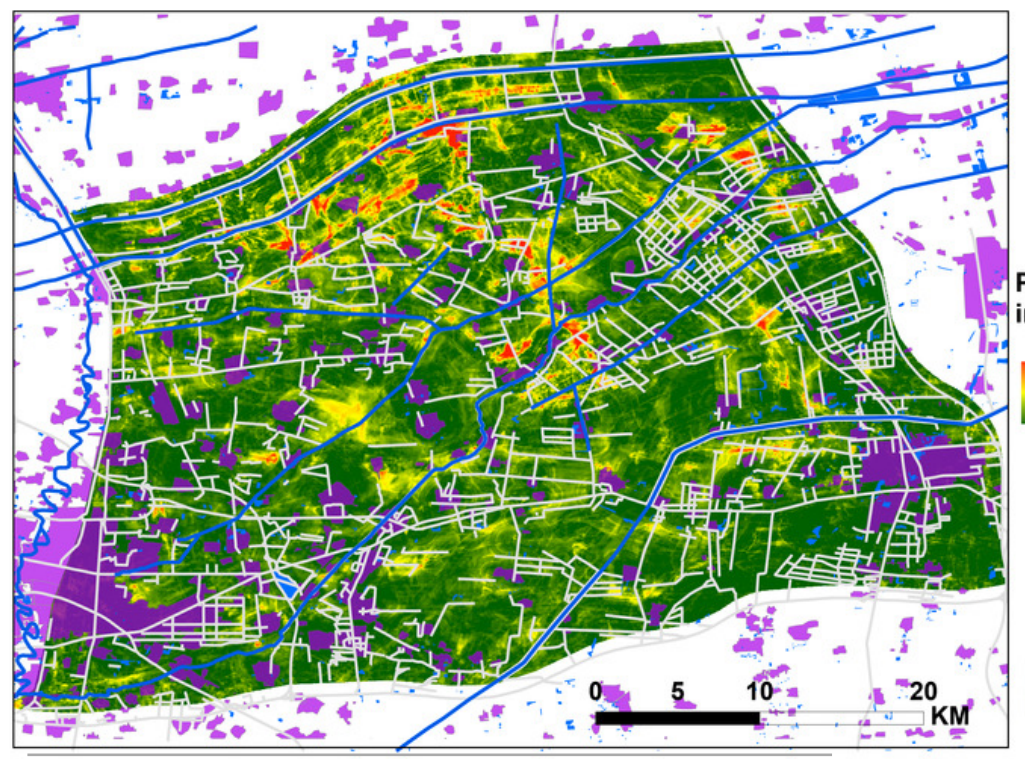

(c) 


\section{Figure 5 (on next page)}

Plots of the relationship between relative index of occurrence (RIO) and observation abundance.

(a) Scatter plot between relative index of occurrence (RIO) and observation abundance; and

(b) partial dependence plot between relative index of occurrence (RIO) and observation abundance (obtained from TreeNet, non-parametric method). 


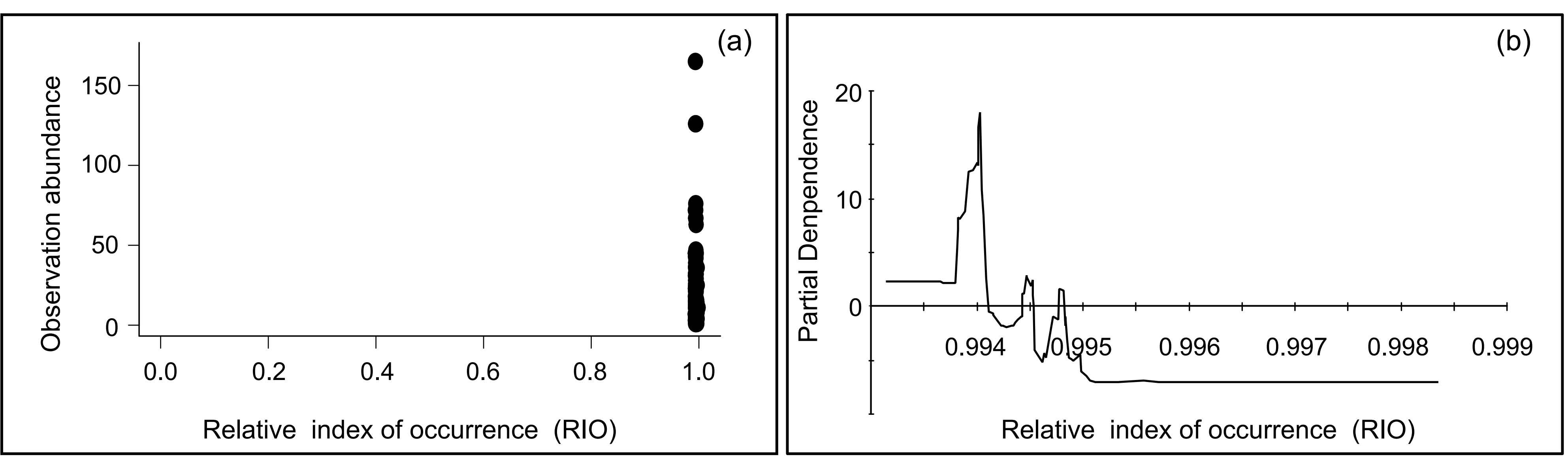

